\title{
Impacts De La Production Du Coton Conventionnel Sur La Qualite Des Eaux Dans Le Bassin Versant De L'alibori Au Benin
}

\author{
Bachabi Alidou Abdoul'ganyi,
}

Laboratoire Pierre PAGNEY : Climat, Eau, Ecosystèmes et Développement (Université d'Abomey-Calavi Cotonou)

\section{Agbon Apollinaire Cyriaque,}

Laboratoire des Applications Géomatiques et Gestion de l'Environnement

(Campus Universitaire d'Adjarra/Université d'Abomey-Calavi

\section{Issa Maman Sanni, Boko Michel,}

Laboratoire Pierre PAGNEY : Climat, Eau, Ecosystèmes et Développement

(Université d'Abomey-Calavi Cotonou)

\section{Resume}

La filière du coton conventionnel est l'une des filières prioritaires du secteur agricole béninois. Cette activité, bien qu'étant l'une des activités agricoles piliers du développement économique béninois, n'est pas sans impacts négatifs sur l'environnement. En effet, les pratiques actuelles des cotonculteurs dans le bassin versant de l'Alibori liées à une mauvaise utilisation des intrants (engrais et pesticides) à des fins agricoles sont sources de dégradation de la qualité des eaux et par conséquence de l'état de santé des populations. La présente recherche analyse des impacts de la production du coton conventionnel sur la qualité des eaux dans le bassin versant de l'Alibori. Les échantillons d'eau ont été prélevés et analysés en saison sèche (décembre 2016) et en saison pluvieuse (août 2017) au niveau de seize (16) sites en eaux de surface, six (6) sites en eaux de puits/forages. Les impacts ont été analysés par la matrice de Léopold et al. (1971) croisée avec le guide, adapté par l'Agence Béninoise pour l'Environnement (ABE, 1998). Ces différentes méthodes utilisées ont été appuyées par les enquêtes socio-économiques sur le terrain. Les résultats montrent la présence des métaux lourds (cadmium et plomb) dans toutes les eaux des puits et de surface en saison pluvieuse (cadmium et le plomb). Ceci s'explique par les apports des produits chimiques par le ruissellement superficiel vers la nappe phréatique et des eaux de surface. Par exemple le cadmium a atteint $60 \mu \mathrm{g} / \mathrm{L}$ alors que la norme est de $5 \mu \mathrm{g} / \mathrm{L}$. 
Selon les résultats d'enquêtes auprès des cotonculteurs, ces métaux lourds absorbés provoquent chez l'homme desmaladies comme les maux de ventre selon $16,67 \%$, les maux des yeux pour $0,34 \%$, la diarrhée par $11,90 \%$, les problèmes de respiration aux dires de $5,78 \%$, la toux pour $3,40 \%$, le rhume selon $2,72 \%$, les infections selon $2,72 \%$, le paludisme selon $14,29 \%$ et les maux de tête par 42,18\%.

Mots clés : Coton conventionnel, Impacts, eaux, bassin versant, Alibori

\title{
Impacts of Conventional Cotton Production on Water Quality in the Alibori to Benin Catchment Area
}

\author{
Bachabi Alidou Abdoul'ganyi, \\ Laboratoire Pierre PAGNEY : Climat, Eau, Ecosystèmes et Développement \\ (Université d'Abomey-Calavi Cotonou) \\ Agbon Apollinaire Cyriaque, \\ Laboratoire des Applications Géomatiques et Gestion de l'Environnement \\ (Campus Universitaire d'Adjarra/Université d'Abomey-Calavi

\section{Issa Maman Sanni, Boko Michel,} \\ Laboratoire Pierre PAGNEY : Climat, Eau, Ecosystèmes et Développement \\ (Université d'Abomey-Calavi Cotonou)
}

\begin{abstract}
The conventional cotton sector is one of the priority sectors of Benin's agricultural sector. This activity, although one of the agricultural activities that are pillars of Benin's economic development, is not without negative impacts on the environment. Indeed, the current practices of cotton growers in the Alibori watershed, linked to the poor use of inputs (fertilisers and pesticides) for agricultural purposes, are a source of degradation of water quality and consequently of the state of health of the populations. This research analyses the impacts of conventional cotton production on water quality in the Alibori catchment area. Water samples were taken and analysed during the dry season (December 2016) and the rainy season (August 2017) at sixteen (16) surface water sites and six (6) well water/borehole sites. The impacts were analysed using the matrix of Léopold et al (1971) crossed with the guide, adapted by the Benin Environment Agency (ABE, 1998). These different methods were supported by socio-economic field surveys. The results show the presence of
\end{abstract}


heavy metals (cadmium and lead) in all well and surface water during the rainy season. This is due to chemical inputs from surface runoff to groundwater and surface water. For example, cadmium has reached $60 \mu \mathrm{g} / \mathrm{L}$ while the standard is $5 \mu \mathrm{g} / \mathrm{L}$. According to the results of surveys of cotton growers, these absorbed heavy metals cause illnesses in humans such as stomach aches (16.67 per cent), eye aches ( 0.34 per cent), diarrhoea ( 11.90 per cent), breathing problems (5.78 per cent), coughs (3.40 per cent), colds (2.72 per cent), infections (2.72 per cent), malaria (14.29 per cent) and headaches (42.18 per cent).

Keywords: Conventional cotton, Impacts, water, watershed, Alibori

\section{Introduction}

L'introduction au Bénin des cultures de rente comme le coton a entrainé des transformations profondes de l'environnement physique et humain. En effet, à la recherche de mieux-être et de la nécessité de satisfaire ses besoins de plus en plus croissants, le producteur agit sur la nature (Kissira, 2010). La culture sur brulis du coton vient au premier rang des spéculations qui obligent les paysans à défricher plusieurs hectares chaque année (Tenté, 2005) avec pour conséquence des transformations profondes de l'environnement physique et humain (AIC, 2009). L'augmentation des emblavures en cotonculture a entraîné la perte de la biodiversité végétale et animale, la dégradation des sols et des eaux (FAO, 2007 et Gomgnimbou et al., 2010). En effet, l'accroissement de la production du coton rythme donc avec le niveau de consommation des pesticides et de dégradation de l'environnement physique et humain (OBEPAB, 2002 ; Kissira, 2010,). Pour Chabi Guiya (2004), l'usage de ces produits comporte des inconvénients au nombre desquels il est à noter la dégradation de l'environnement et de l'état de santé des populations. Aussi, l'utilisation massive des engrais et insecticides chimiques cause-t-elle des dommages environnementaux et sanitaires: appauvrissement des terres, pollution des points d'eaux, puis engendre des problèmes de santé chez les populations (Max Havelaar, 2005). Le présent article analyse les impacts de la production du coton conventionnel sur la qualité des eaux dans le bassin versant de l'Alibori et donc sur la santé des populations. Ce milieu s'étend sur trois (03) départements (Alibori, Atacora, et Borgou) et couvre neufs (09) Communes : Alibori (Kandi, Banikoara, Gogounou, Malanville et Karimama), Atacora (Kérou, Péhonco), Borgou (Sinendé, Bemberèkè). Selon les données de IGN (1963) et DGEau (2008) le bassin versant de l'Alibori est situé entre $10^{\circ} 12^{\prime}$ et $12^{\circ} 00^{\prime}$ de latitude nord et entre $1^{\circ} 48^{\prime}$ et $3^{\circ} 18^{\prime}$ de longitude est (figure 1 ). 


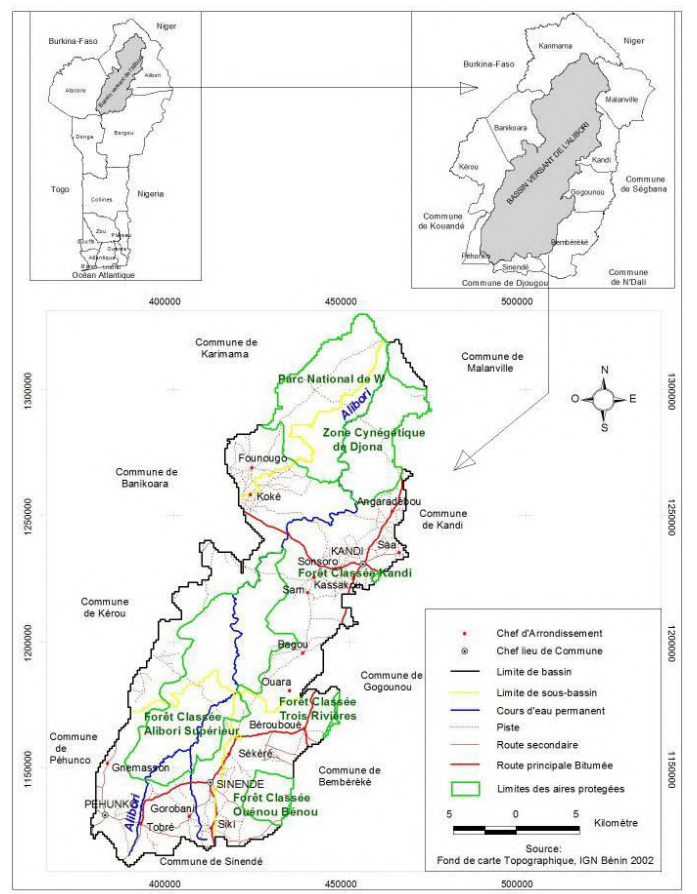

Figure 8 : Situation géographique et administrative du bassin versant de l'Alibori

\section{Matériels et méthodes}

\subsection{Nature et sources données utilisées}

Plusieurs types de données ont servi à la réalisation de cet article. Il s'agit :

- des données relatives aux mesures des paramètres physicochimiques des échantillons des eaux du bassin versant de l'Alibori, collectées sur deux périodes :une période sèche (décembre 2016) et une autre période humide (août 2017) ;

- les données et informations sur la production du coton conventionnel et leurs effets néfastes sur l'eau et la santé des populations du bassin versant de l'Alibori et les stratégies d'adaptation paysannes recueillies auprès des populations enquêtées et au cours des observations de terrain.

\subsection{Collecte des données}

La recherche documentaire a été réalisée au niveau des institutions de recherche dont les champs d'activité sont en liaison avec l'objet de la présente recherche. En dehors des centres locaux de documentations, plusieurs sites WEB spécialisés ont également été consultés dans le cadre de la documentation. 
Les enquêtes de terrain sont effectuées dans toutes les neuf (09) communes du bassin versant de l'Alibori. Les différentes catégories de groupes cibles sont constituées de ménages de cotonculteurs. Les autres personnes ressources (personnels du SCDA, encadreurs retraités, responsables AIC, etc.) ont été choisies en fonction de leur responsabilité dans la cotonculture dans leur localité respective. La Méthode d'Analyse Multicritère a été utilisée pour choisir les arrondissements où les enquêtes sont menées.

Cette méthode a consisté à apprécier le niveau de pertinence du choix de chaque arrondissement en tenant compte du niveau de production cotonnière et de la proximité du cours d'eau de l'Alibori (l'eau constitue un élément à travers lequel on peut apprécier le niveau de pollution/dégradation d'un milieu).

La taille de l'échantillon est déterminée par le protocole statistique de Schwartz (1995) dont la formule est la suivante : $\mathrm{X}=\mathrm{Z} \alpha^{2} \mathrm{Pq} / \mathrm{d}^{2}$. Le tableau I présente l'effectif des cotonculteurs interrogés par commune.

Tableau VII: : Effectif des ménages agricoles par Commune dans le bassin versant de l'Alibori

\begin{tabular}{|l|l|l|l|l|}
\hline Communes & $\begin{array}{l}\text { Ménages } \\
\text { agricoles (2013) }\end{array}$ & $\begin{array}{l}\text { Nombre villages par } \\
\text { commune }\end{array}$ & $\begin{array}{l}\text { Nombre villages } \\
\text { enquêtés }\end{array}$ & $\begin{array}{l}\text { Taille de } \\
\text { l'échantillon }\end{array}$ \\
\hline Pehonco & 7021 & 26 & 7 & 25 \\
\hline Kérou & 9610 & 28 & 2 & 29 \\
\hline Sinendé & 6601 & 35 & 6 & 26 \\
\hline Bemberèkè & 11690 & 42 & 7 & 35 \\
\hline Gogounou & 9581 & 34 & 7 & 31 \\
\hline Kandi & 15944 & 48 & 6 & 49 \\
\hline Banikoara & 17166 & 69 & 6 & 54 \\
\hline Malanville & 15250 & 31 & 2 & 43 \\
\hline Karimama & 8727 & 18 & & 31 \\
\hline Total & $\mathbf{1 0 1 5 9 0}$ & $\mathbf{3 3 1}$ & $\mathbf{4 3}$ & $\mathbf{3 2 3}$ \\
\hline
\end{tabular}

Source: INSAE, 2013 et traitement des données, septembre 2016

A la lecture du tableau I, 323 chefs ménages agricoles sont interrogés sur un total de 101590 . En plus des chefs ménages agricoles qui sont ciblés, les entretiens ont lieu avec des acteurs (109 personnes) des institutions (AIC, CAGIA, CCPC, SCDA, ONG, OP, élus locaux etc.) impliquées dans la production cotonnière.

\subsection{Méthodes de Prélèvements des échantillons d'eau}

Les échantillons d'eau ont été prélevés, suivant les protocoles décrits par Claasen (1982), Barcelona et al., (1985), Hounslow (1995), Akognongbé (2014) en saison pluvieuse (août) et en saison sèche (décembre) au niveau de seize (16) sites en eaux de surface, six (6) sites en eaux de puits/forages. Ces sites ont été choisis en tenant compte des critères tels que la topographie, le sens de l'écoulement des eaux, la répartition géographique au niveau 
communal et l'accessibilité aux sites. Les points de prélèvements, géoréférencés à l'aide du Global Positionning System (GPS) sont répartis dans le bassin versant de l'Alibori comme l'illustre la figure 2.

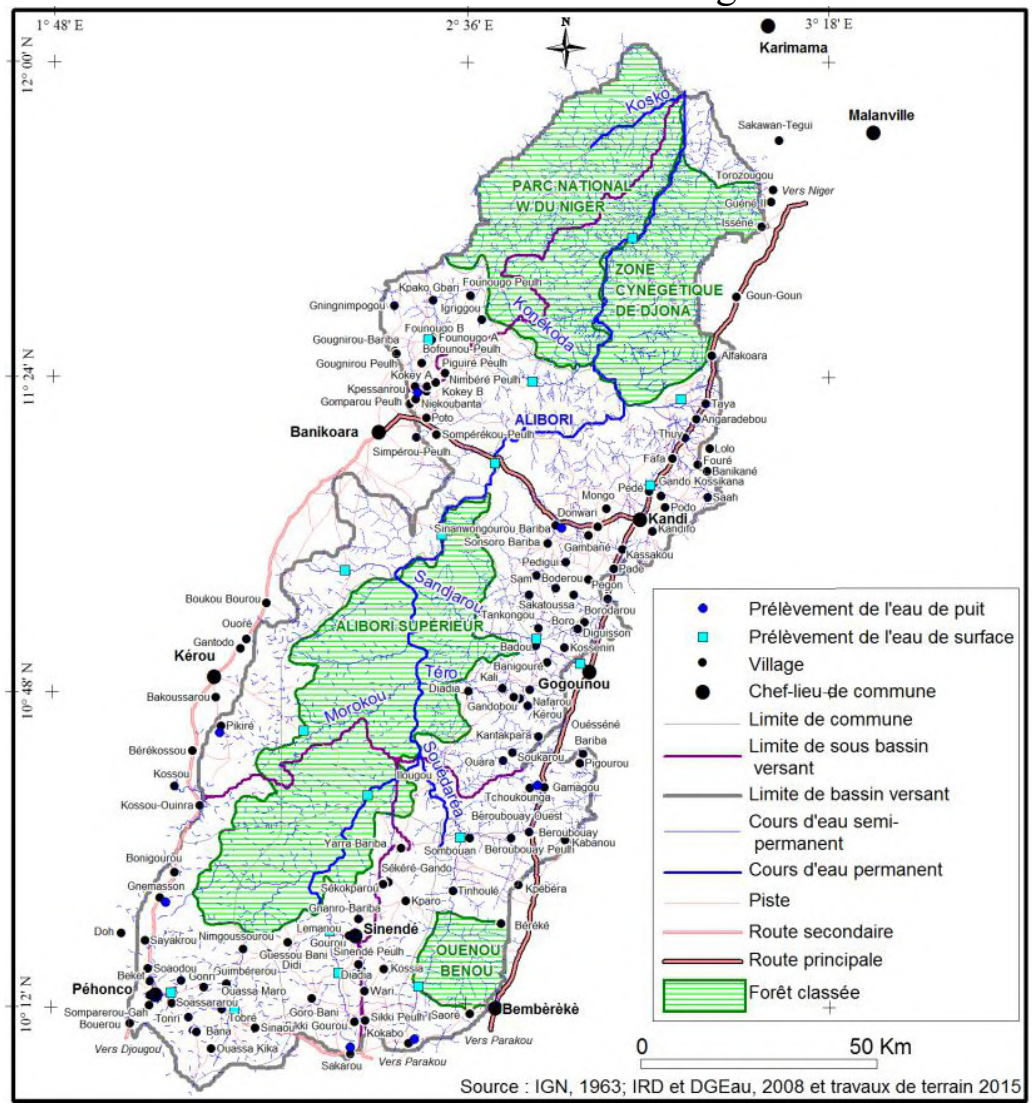

Figure 9: Points de prélèvements des échantillons d'eaux et mesures de certains paramètres (température, $\mathrm{pH}$ et conductivité)

Les eaux sont prélevées avec toutes les précautions d'asepsie nécessaires au moyen des bouteilles en plastique d'eau minérale de capacité 1,5 L pour les analyses physico-chimiques. Chaque bouteille est rincée par l'eau qu'elle doit contenir. Il est ensuite conservé à une température de $4{ }^{\circ} \mathrm{C}$. Une fois les prélèvements faits, les bouteilles contenant des échantillons sont soigneusement étiquetées et conservées au frais dans des matériels appropriés (glacières chargées en permanence de la glace ou de stabilisateurs) pour éviter les réactions chimiques qui peuvent se produire dans la bouteille et modifier la composition de l'eau prélevée. Ces échantillons sont ensuite immédiatement acheminés au laboratoire pour les analyses. Conjointement à tout échantillonnage, la prise de mesures de certains paramètres de l'eau tels que la conductivité, l'oxygène dissout, le $\mathrm{pH}$ et la température a été effectuée directement sur le site. Ces différentes analyses ont été faites au Laboratoire d'Hydrologie Appliquée (LHA) de l'Université d'Abomey-Calavi. Les 
analyses de ces échantillons d'eau ont permis d'apprécier la qualité physicochimique et bactériologique des eaux de consommation en se référant aux normes physico-chimiques définies par l'OMS et appliquées au Bénin. Les eaux ont été qualifiées de mauvaise qualité, lorsqu'une valeur obtenue dépasse la norme maximale fixée pour ce paramètre. La Planche 1 montre des prélèvements d'échantillons d'eau de rivière et de forage dans le bassin versant de l'Alibori pour analyse.
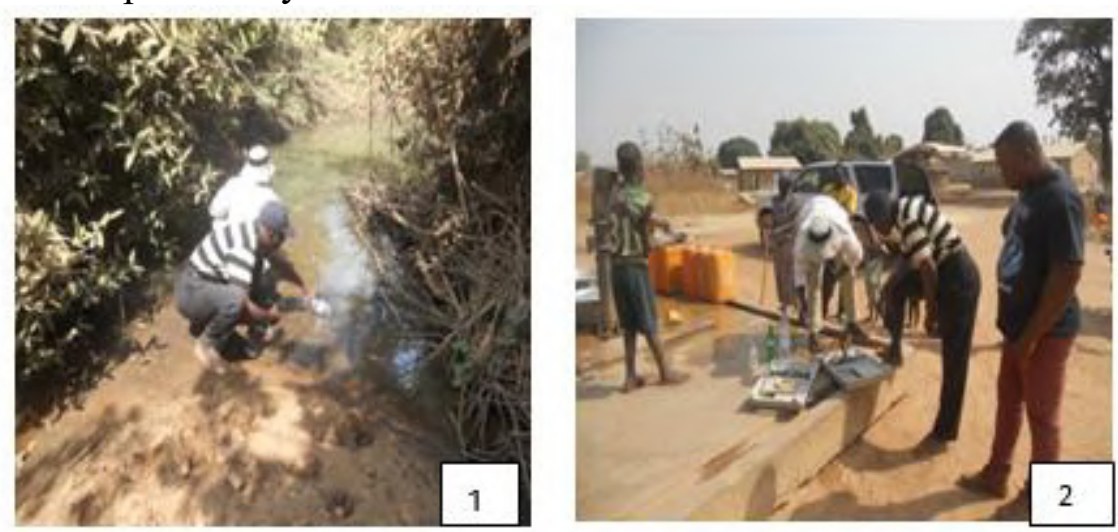

Planche 1: Prélèvements d'échantillons d'eau de rivière (Photo 1) et de forage (Photo 2) pour analyse

Prise de vues: Bachabi, décembre 2016

Le tableau II suivant présente les paramètres physico-chimiques d'échantillon d'eau de surface et des puits/forages mesurés au niveau de quelques sites du bassin versant de l'Alibori.

Tableau VIII: Paramètres physico-chimiques des échantillons d'eau mesurés

\begin{tabular}{|c|c|c|c|}
\hline PARAMETRES & SYMBOLES & $\begin{array}{l}\text { METHODES } \\
\text { UTULISEES }\end{array}$ & NORMES \\
\hline Température $\left({ }^{\circ} \mathbf{C}\right)$ & $\mathbf{T}$ & Electrometrique & - \\
\hline Potentiel d'Hydrogène & $\mathrm{pH}$ & Electrometrique & Entre 6,5 et 8,5 \\
\hline Conductivité Electrique & $\mathrm{CE}$ & Electrometrique & $250 \mathrm{us} / \mathrm{cm}$ \\
\hline Turbidité & NTU & Colorimetrique & 5 \\
\hline Couleur & PtCo & Colorimetrique & 15 \\
\hline Nitrates $(\mathrm{mg} / \mathrm{L})$ & NO3 & $\begin{array}{c}\text { Réduction au } \\
\text { cadmium }\end{array}$ & $45 \mathrm{mg} / \mathrm{L}$ \\
\hline Nitrites $(\mathrm{mg} / \mathrm{L})$ & $\mathrm{NO} 2$ & Diazotation & $3,2 \mathrm{mg} / \mathrm{L}$ \\
\hline $\begin{array}{c}\text { Azote Total Kjeldahl } \\
(\mathrm{mg} / \mathrm{L})\end{array}$ & NTK & Méthode Nessler & $1 \mathrm{mg} / \mathrm{L}$ \\
\hline Orthophosphate $(\mathrm{mg} / \mathrm{L})$ & PO4 & $\begin{array}{l}\text { Méthode Acide } \\
\text { ascorique }\end{array}$ & $5 \mathrm{mg} / \mathrm{L}$ \\
\hline Ammonium (mg/L) & NH4 & Méthode Salicylate & $0,5 \mathrm{mg} / \mathrm{L}$ \\
\hline Cuivre (mg/L) & $\mathrm{Cu}$ & $\begin{array}{c}\text { Méthode } \\
\text { Bicinchoninate }\end{array}$ & $2 \mathrm{mg} / \mathrm{L}$ \\
\hline
\end{tabular}




\begin{tabular}{|c|c|c|c|}
\hline Zinc $(\mathbf{m g} / \mathbf{L})$ & $\mathrm{Zn}$ & Méthode Zinco & $3 \mathrm{mg} / \mathrm{L}$ \\
\hline Cadmium $(\boldsymbol{\mu g} / \mathbf{L})$ & $\mathrm{Cd}$ & Méthode Ditizone & $5 \mu \mathrm{g} / \mathrm{L}$ \\
\hline Plomb $(\boldsymbol{\mu g} / \mathbf{L})$ & $\mathrm{Pb}$ & Méthode Extraction & $50 \mu \mathrm{g} / \mathrm{L}$ \\
\hline
\end{tabular}

\subsection{Méthodes d'analyse des échantillons d'eau prélevés}

Le traitement et l'exploitation des données nécessitent d'outils adéquats notamment le logiciel diagramme et la grille d'évaluation du Système d'Evaluation de la Qualité des eaux (SEQ Eau). Il importe de préciser que, c'est la grille d'évaluation SEQ Eau ou grille multi-usage qui a été utilisée pour l'analyse des données en vue d'apprécier la qualité des eaux prélevées.

\section{- Le logiciel diagramme}

C'est un logiciel d'hydrochimie en distribution libre facilitant l'exploitation d'analyse d'eau. Ces fonctions sont variées et complètes, en voici quelquesunes :

* les diagrammes: - Piper ; Stiff ; stabler ; Schoeller-Berkalov ; Binaire ; Korjinski ; Rose des vents ;

* les paramètres calculés : balances ioniques; teneurs en cations; dureté ; teneurs en anions ;

indice de saturation (calcite, dolomite, aragonite, gypse).

De ce fait, les diagrammes de Piper et de Schoeller-Berkalov ont été utilisés dans le cadre de cette recherche (figure 3 et 4 ).

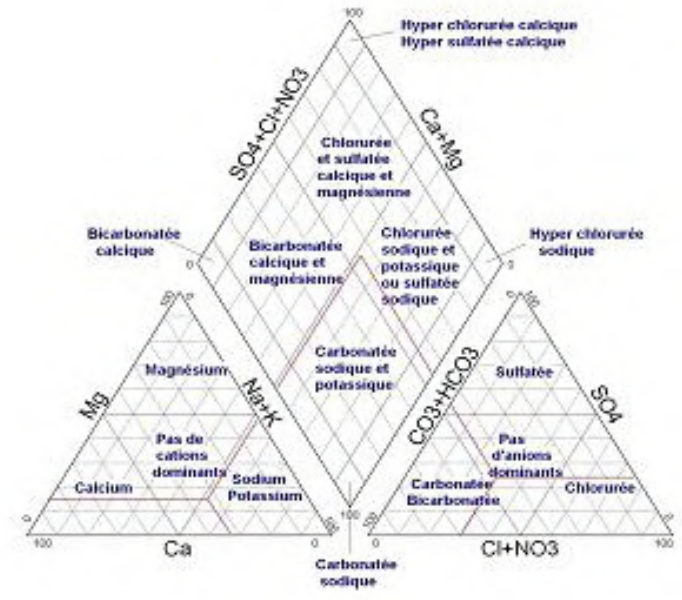

Figure 3: Diagramme Piper

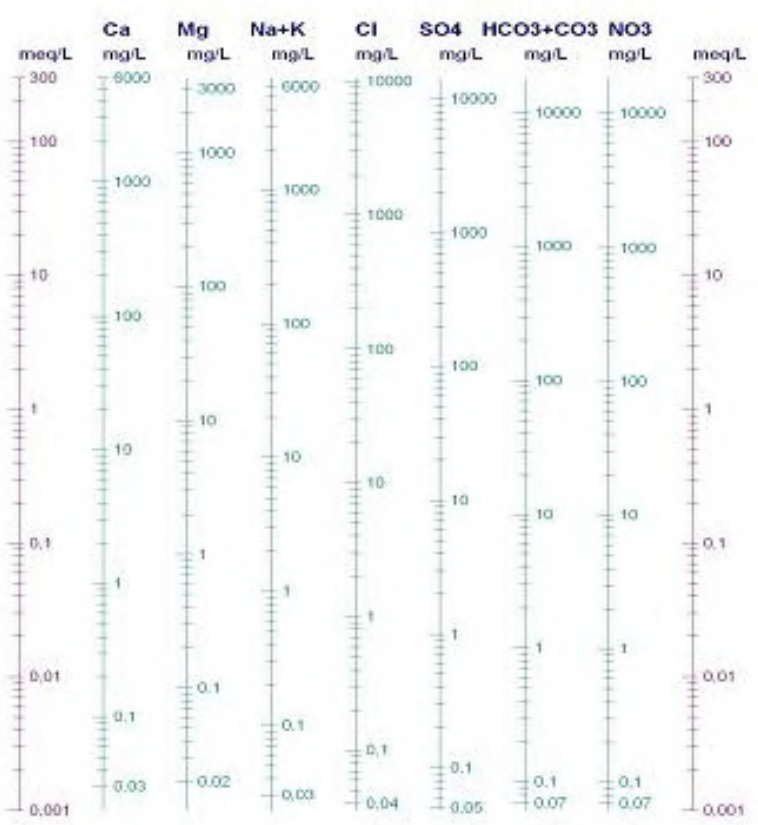

Figure 4: Diagramme de Schoeller-Berkalov 


\section{○ La grille d'évaluation SEQ Eau}

L'application d'Evaluation de la Qualité des eaux de surface, SEQEau, permet l'importation des données relatives à un prélèvement et l'évaluation de la qualité de l'eau par le calcul d'indices et de classes de qualité par l'altération et de classes d'aptitude aux fonctions et usages. L'application permet de visualiser les planches de calcul selon plusieurs critères de sélection

- calcul des indices et classes de qualité par prélèvement pour une altération ;

- calcul des classes d'aptitude de l'eau aux usages et fonctions par prélèvement;

- calcul des données élaborées en une station pour deux (2) années complètes consécutives ;

- calcul sur plusieurs stations, plusieurs années, en sélectionnant une ou plusieurs altérations et un ou plusieurs usages et fonctions ;

- calcul des chronologies de données élaborées par prélèvement ou par période de plusieurs années complètes consécutives et affichage graphique permettant jusqu'à 5 données élaborées par graphique ;

- calcul des profils en long de données élaborées pour une période de 2 années complètes consécutives et affichage graphique permettant jusqu'aux données élaborées par graphique ;

- calcul de la concentration objectif à partir des classes d'aptitude à une fonction ou à un usage.

Les analyses de ces échantillons d'eau ont permis d'apprécier la qualité physico-chimique des eaux de consommation en se référant aux normes physico-chimiques définies par l'OMS et appliquées au Bénin. Les eaux ont été qualifiées de mauvaise qualité, lorsqu'une valeur obtenue dépasse la norme maximale fixée pour ce paramètre.

\subsection{Méthode d'identification des impacts environnementaux et sociaux de la production du coton conventionnel dans le bassin versant de l'Alibori \\ L'utilisation de la matrice de type Léopold et al. (1971) est présentée dans le tableau III et a permis d'identifier les composantes environnementales et sociales affectées. Ensuite, il a été question de croiser les mutations observées avec ces composantes dans le bassin versant de l'Alibori.}


Tableau III: Matrice d'identification des impacts sur les composantes de l'environnement

\begin{tabular}{|c|c|c|c|}
\hline Activités & \multicolumn{3}{|c|}{ Composantes environnementales } \\
\hline \multirow{3}{*}{$\begin{array}{l}\text { Production } \\
\text { du coton }\end{array}$} & Milieu physique & $\begin{array}{l}\text { Milieu } \\
\text { biologique }\end{array}$ & Milieu humain \\
\hline & Eau & Faune & Santé \\
\hline & Qualité & $\begin{array}{l}\text { Dynamique } \\
\text { de la faune } \\
\text { aquatique }\end{array}$ & $\begin{array}{l}\text { Dégradation de } \\
\text { la santé des } \\
\text { cotonculteurs }\end{array}$ \\
\hline
\end{tabular}

Source : Adapté de l’ABE (1998)

La clarification conceptuelle qui est utilisée dans le cadre de cette évaluation s'est référée aux définitions qui tiennent compte de la nature de l'impact (direct ou indirect), de son étendue (ponctuelle, locale ou générale) et de sa durée (temporaire ou permanente). Le critère de l'intensité de l'impact positif (+) ou négatif (-) qualifiée de :

- forte $[+++]$, quand la qualité de l'élément est améliorée dans son ensemble, de façon significative ;

- forte [---], quand l'élément est atteint dans son ensemble au point où sa qualité est considérée altérée de façon irréversible ;

- moyenne [++], quand la qualité de l'élément est améliorée moyennement ;

- moyenne [--], quand l'élément est atteint mais pas dans son ensemble ou de façon irréversible ;

- faible [+], quand la qualité de l'élément est améliorée de façon marginale et sur une courte durée ;

- faible [-], quand l'élément n'est atteint que de façon marginale et sur une courte durée. [-] : l'impact peut être atténué.

Par ailleurs, l'évaluation de l'importance des impacts repose sur une méthodologie qui intègre les trois (3) paramètres à savoir la durée (momentanée, temporaire ou permanente), l'étendue (ponctuelle, locale ou régionale) et le degré de perturbation (très fort, fort, moyen ou faible) de l'impact négatif. Le croisement de ces trois (3) paramètres à travers le cadre de référence de l'ABE a permis de déduire si l'importance de l'impact est forte, moyenne ou faible. Ce cadre de référence d'évaluation de l'importance des impacts fonctionne avec les critères que sont : la durée, l'étendue et le degré de perturbation. Ces critères sont d'égale pondération (tableau IV). 
Tableau IV : Cadre de référence pour l'évaluation de l'importance des impacts

\begin{tabular}{|c|c|c|c|c|c|}
\hline \multirow[t]{3}{*}{ Durée } & \multirow[t]{3}{*}{ Etendue } & \multicolumn{4}{|c|}{ Degré de perturbation } \\
\hline & & & Moyenne & Forte & Très forte \\
\hline & & \multicolumn{4}{|c|}{ Importance de l'impact } \\
\hline Momentanée & Ponctuelle & Moyenne & Moyenne & Moyenne & Forte \\
\hline Momentanée & Locale & Moyenne & Moyenne & Forte & Forte \\
\hline Temporaire & Ponctuelle & Moyenne & Moyenne & Forte & Très forte \\
\hline Temporaire & Locale & Moyenne & Moyenne & Forte & Très forte \\
\hline Momentanée & Régionale & Moyenne & Forte & Forte & Très forte \\
\hline Permanente & Ponctuelle & Moyenne & Forte & Forte & Très forte \\
\hline Temporaire & Régionale & Moyenne & Forte & Très forte & Très forte \\
\hline Permanente & Locale & Moyenne & Forte & Très forte & Très forte \\
\hline Permanente & Régionale & Forte & Très forte & Très forte & Très forte \\
\hline
\end{tabular}

Source : Adapté de l'ABE (1998)

\section{Résultats}

\subsection{Analyses des paramètres physico-chimiques dans le bassin versant}

\section{de l'Alibori}

\subsubsection{Analyses des paramètres physico-chimiques des échantillons d'eau} des puits/Forages

Les principaux micropolluants impliqués dans les phénomènes de pollutions chimiques sont les métaux lourds (cadmium, plomb, cuivre, zinc, etc.) et certains composés minéraux toxiques. La figure 5 présente les résultats d'analyse des paramètres physico-chimiques des métaux (Cadmium, Plomb, Cuivre, Zinc) en saison sèche et en saison pluvieuse au niveau des eaux des sources d'eau (puits ou forages).
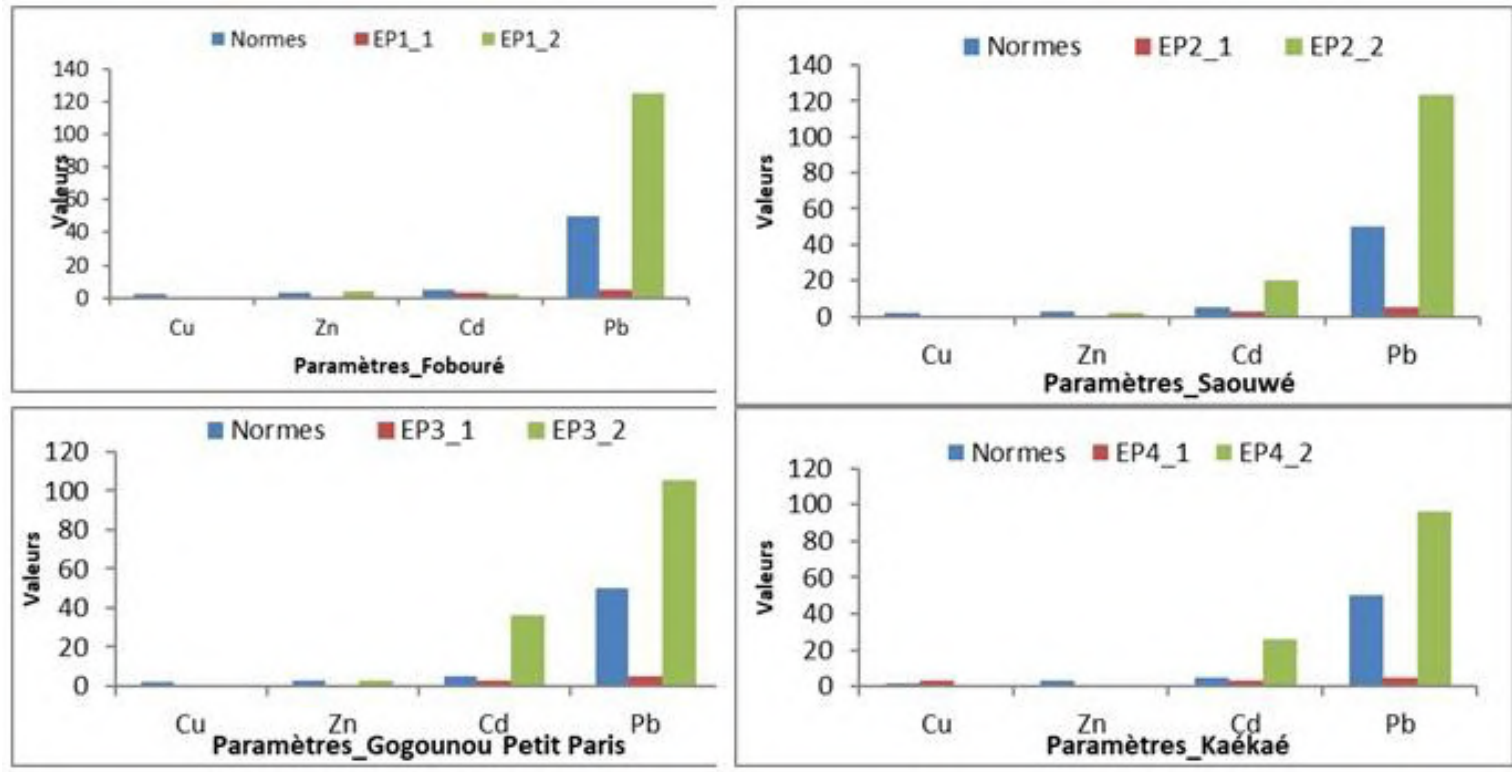

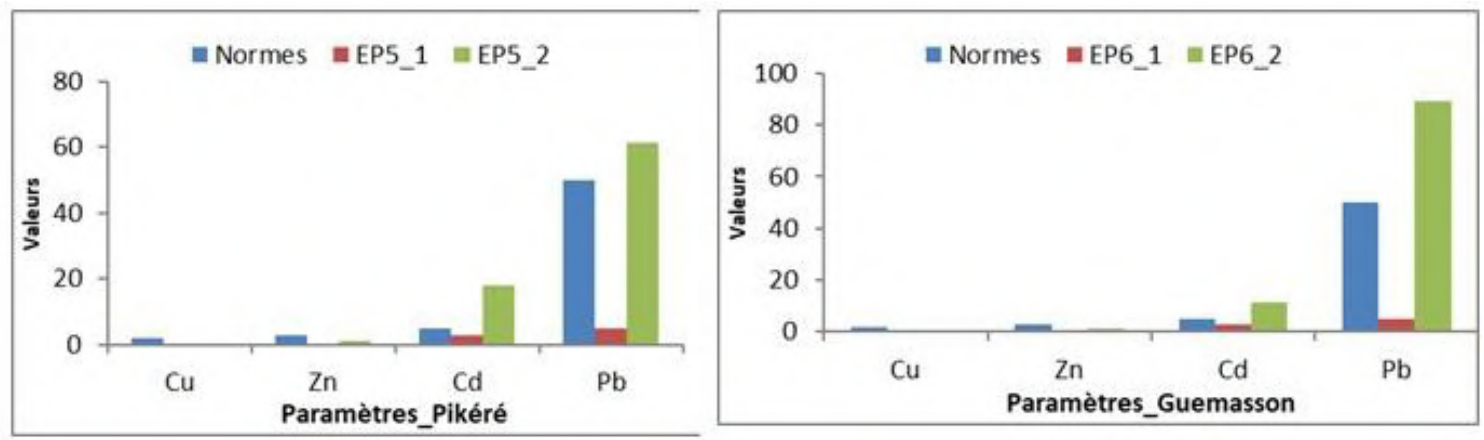

Figure 5 : Etat d'évolution des métaux lourds dans les puits/forages du bassin versant cotonnier de l'Alibori

Source des données : Résultats d'Analyse physico-chimique du LHA, décembre 2016 et août 2017

\section{Légende :}

EP 1-1 : Prélèvement du site 1 en saison sèche ; EP 1-2 : Prélèvement du site 1 en saison pluvieuse ; EP 2-1: Prélèvement du site 2 en saison sèche ; EP 2-2: Prélèvement du site 2 en saison pluvieuse ; EP 3-1 : Prélèvement du site 3 en saison sèche ; EP 3-2 : Prélèvement du site 3 en saison pluvieuse ; EP 4-1 : Prélèvement du site 4 en saison sèche ; EP 4-2 : Prélèvement du site 4 en saison pluvieuse ; EP 5-1 : Prélèvement du site 5 en saison sèche ; EP 5-2 : Prélèvement du site 5 en saison pluvieuse ; EP 6-1 : Prélèvement du site 6 en saison sèche ; EP 6-2 : Prélèvement du site 6 en saison pluvieuse.

De l'analyse de cette figure 5, il ressort qu'une accumulation des métaux lourds (cadmium, plomb, cuivre, zinc) dans toutes les eaux des puits en saison pluvieuse. La présence de ces métaux lourds dans les eaux pourrait s'expliquer par les apports des produits chimiques par le ruissellement superficiel vers la nappe phréatique dans la mesure où l'utilisation des intrants chimiques est faite en saison pluvieuse. La concentration de ces métaux lourds, en saison pluvieuse, est largement supérieure à la Valeur Maximale Admissible (VMA) admise par l'OMS dans tous les points d'eau analysés. Les cycles biogéochimiques des éléments métalliques ainsi que des espèces organiques et inorganiques sont influencés à la fois par les phénomènes naturels et par les activités humaines (Bloundi, 2005). Par contre, pour les points d'eau des puits/forages analysée en saison sèche, l'accumulation des métaux lourds est largement en dessous de la Valeur Maximale Admissible (VMA) admise par l'OMS.

\subsubsection{Analyses des paramètres physico-chimiques des échantillons d'eau de surface}

Le tableau $\mathrm{V}$ présente les résultats des analyses physico-chimiques des échantillons d'eau de surface en saison sèche au niveau de 16 localités du bassin versant de l'Alibori. 
Tableau V : Résultats d'analyse physico-chimique des échantillons d'eau de surface en saison sèche

\begin{tabular}{|c|c|c|c|c|c|c|c|c|c|c|c|c|c|c|c|c|c|c|c|}
\hline Paramètres & symboles & \begin{tabular}{|l|} 
Méthodes \\
utilisées
\end{tabular} & Normes & \multicolumn{16}{|c|}{ ECHANTILLONS D'EAUX } \\
\hline Cuivre (mg/L) & $\mathrm{Cu}$ & $\begin{array}{l}\text { Méthode } \\
\text { Bicinchoninate }\end{array}$ & $2 \mathrm{mg} / \mathrm{L}$ & $<0,01$ & 0,02 & 0,04 & 0,13 & 0,06 & 0,05 & 0,03 & 0,03 & 2,97 & 0,01 & 0,11 & 0,03 & 0,37 & 0,17 & 0,1 & 0,03 \\
\hline Zinc (mg/L) & $\mathrm{Zn}$ & Methode Zinco & $3 \mathrm{mg} / \mathrm{L}$ & 0,17 & 0,07 & $<0,01$ & 0,02 & 0,07 & 0,06 & 0,02 & 0,04 & 0,14 & 0,09 & 0,07 & 0,09 & 0,1 & 0,07 & 0,1 & 0,1 \\
\hline Plomb $(\mu \mathrm{g} / \mathrm{L})$ & $\mathrm{Pb}$ & $\begin{array}{l}\text { Méthode } \\
\text { Extraction }\end{array}$ & $50 \mu \mathrm{g} / \mathrm{L}$ & 8 & $<5$ & 4 & 5 & 8 & $<5$ & 5 & 7 & $<5$ & $<5$ & 5 & 5 & $<5$ & $<5$ & $<5$ & $<5$ \\
\hline
\end{tabular}

Source: Résultats d'analyse LHA, décembre 2016

\section{Légende}

E1 : Sinendé ; E2 : Bembèrèkè ; E3 : Yara Sombouan ; E4 : Kandi Pédé ; E5 : Sassarou ; E6 :

Koufonou ; E7 : Goundarou ; E8 : Takongou ; E9 : Sinanwangourou ; E10 : Banikoara ;

E11 : Founougo ; E12 : Gbassa ; E13 : Pikéré ; E14 : Gnémassoun ; E15 : Ouassa Puhunco ;

E16 : Tobré

\section{Inférieur à la Norme \\ Norme \\ Supérieur à la Norme}

L'examen du tableau $\mathrm{V}$ montre qu'en dehors des eaux de surface de Sinanwangourou (E9) où la valeur du Cuivre $(2,97 \mathrm{mg} / \mathrm{L})$ est supérieure à la Norme $(2 \mathrm{mg} / \mathrm{L})$, les valeurs des paramètres Cuivre, Zinc, Cadmium et Plomb sont conformes aux normes en saison sèche. En effet, seule la teneur en Cuivre des eaux de surface au niveau de Sinanwangourou (E9), comparée au seuil recommandé (Centrations Maximales Admissibles (CMA) fixées par l'OMS), indique un niveau de pollution des eaux de surface; les eaux de surface des autres villages présentent une teneur en Cuivre en dessous de la norme fixée.

Le tableau VI présente les résultats des analyses physico-chimiques des échantillons d'eau de surface en saison pluvieuse au niveau de 16 localités du bassin versant de l'Alibori. 
Tableau IXI: Résultats d'analyse physico-chimique des échantillons d'eau de surface en saison pluvieuse

\begin{tabular}{|c|c|c|c|c|c|c|c|c|c|c|c|c|c|c|c|c|c|c|c|}
\hline Paramètre & $\begin{array}{l}\text { Sym- } \\
\text { boles }\end{array}$ & $\begin{array}{l}\text { Méthodes } \\
\text { utilisées }\end{array}$ & NORME & \multicolumn{16}{|c|}{ ECHANTILLONS D'EAUX } \\
\hline & & & & E1 & E2 & E3 & E4 & E5 & E6 & E7 & E8 & E9 & E10 & E11 & E12 & E13 & E14 & E15 & E16 \\
\hline $\begin{array}{l}\text { Cuivre } \\
(\mathrm{mg} / \mathrm{L})\end{array}$ & $\mathrm{Cu}$ & $\begin{array}{c}\text { Méthode } \\
\text { Bicinchoninate }\end{array}$ & $2 \mathrm{mg} / \mathrm{L}$ & 0,10 & 0,18 & 0,27 & 0,22 & 0,04 & 0,358 & 0,055 & 2,46 & $<0,00$ & $<0,001$ & 0,098 & $<0,00$ & $<0,001$ & 0,036 & 0,176 & 0,186 \\
\hline $\begin{array}{c}\text { Zinc } \\
(\mathrm{mg} / \mathrm{L})\end{array}$ & $\mathrm{Zn}$ & $\begin{array}{c}\text { Methode } \\
\text { Zinco }\end{array}$ & $3 \mathrm{mg} / \mathrm{L}$ & 1,16 & 1,61 & 1,86 & 1,46 & 2,72 & 1,15 & 1,14 & 2,09 & 0,77 & 1,31 & $\mathbf{0 , 7 3}$ & 1,21 & 0,96 & 1,17 & 1,4 & 0,74 \\
\hline $\begin{array}{c}\text { Cadmium } \\
(\mu \mathrm{g} / \mathrm{L})\end{array}$ & $\mathrm{Cd}$ & $\begin{array}{l}\text { Methode } \\
\text { Ditizone }\end{array}$ & $5 \mu \mathrm{g} / \mathrm{L}$ & 20,3 & 60,3 & 17,1 & 19,3 & 11,8 & 42,5 & 18,13 & 11,56 & 14,38 & 16,25 & 17,81 & 22,81 & 22,5 & 8,13 & 10,94 & 21,56 \\
\hline $\begin{array}{l}\text { Plomb } \\
(\mu \mathrm{g} / \mathrm{L})\end{array}$ & $\mathrm{Pb}$ & $\begin{array}{l}\text { Methode } \\
\text { Extraction }\end{array}$ & $50 \mu \mathrm{g} / \mathrm{L}$ & 110 , & 115 , & 143 , & 306 , & 214 & 172,5 & 116,5 & 108,1 & 99 & 150,9 & 64 & 62,8 & 75,31 & 85 & 98,44 & 97,81 \\
\hline
\end{tabular}

Source: Résultats d'analyse LHA, aô̂t 2017

\section{Légende}

E1 : Sinendé ; E2 : Bembèrèkè ; E3 : Yara Sombouan ; E4 : Kandi Pédé ; E5 : Sassarou ; E6 : Koufonou ; E7 : Goundarou ; E8 : Takongou ; E9 : Sinanwangourou ; E10 : Banikoara ; E11 : Founougo ; E12 : Gbassa ; E13 : Pikéré ; E14 : Gnémassoun ; E15 : Ouassa Puhunco ; E16 : Tobré

\section{\begin{tabular}{|c|c|c|}
\hline $\begin{array}{c}\text { Inférieur à la Norme } \\
\text { Norme }\end{array}$ \\
Supérieur à la Norme
\end{tabular}}

Il ressort qu'en saison pluvieuse, il ressort une forte accumulation des métaux lourds comme le Cadmium et le Plomb dans toutes les eaux de surface prélevées (tableau VI). Ainsi, les concentrations importantes de métaux lourds (Cadmium et le Plomb) dans les eaux de surface en saison pluvieuse seraient liées à l'utilisation des intrants chimiques pour la production du coton conventionnel. En effet, les produits chimiques sont envoyés par le ruissellement superficiel vers les cours d'eau. Par contre, les valeurs du Zinc et de Cuivre présentes dans ces eaux de surface au cours de la même saison pluvieuse sont largement en dessous des normes fixées par l'OMS à l'exception de Takongou (E8) où la valeur du Cuivre $(2,46 \mathrm{mg} / \mathrm{L})$ est légèrement supérieure à la norme $(2 \mathrm{mg} / \mathrm{L})$. Ce contraste constaté entre le $1^{\mathrm{er}}$ prélèvement-saison sèche et le $2^{\text {ème }}$ prélèvement-saison des pluies pourrait s'expliquer par un lessivage des produits d'épandage et leur transport par le ruissellement, des champs vers les cours d'eau.

\subsection{Impacts sanitaires de la production du coton conventionnel}

Les métaux lourds absorbés chez l'homme, s'accumulent spécifiquement dans différents organes (les reins, le foie et même le cerveau) et provoquent des troubles divers et variés propres à chaque métal comme le montre le tableau VII ci-dessous. 
Tableau VII : Quelques impacts des métaux lourds sur la santé humaine

\begin{tabular}{|c|c|}
\hline Particules & Toxicité \\
\hline Plomb & $\begin{array}{c}\text { Troubles du système nerveux, affection } \\
\text { du foie et des reins }\end{array}$ \\
\hline Cadmium & Affections respiratoires, troubles rénaux \\
\hline Mercure & $\begin{array}{c}\text { Troubles du système nerveux (mémoire, } \\
\text { fonctions sensorielles de coordination) }\end{array}$ \\
\hline Nickel & $\begin{array}{c}\text { Maladies respiratoires, asthme, } \\
\text { malformations congénitales, cancers }\end{array}$ \\
\hline
\end{tabular}

Source: Di Benedetto M., 1997, Adam S., 2012 et enquêtes de terrain, septembre 2016

L'examen du tableau VII montre que les métaux lourds provoquent des troubles divers et variés sur la santé humaine. Il s'agit entre autres, en ce qui concerne le Cadmium et le Plomb: des troubles du système nerveux, des affections du foie et des reins, les affections respiratoires, des troubles rénaux, etc. La figure 4 montre les proportions des principales maladies liées à la culture du coton conventionnel.
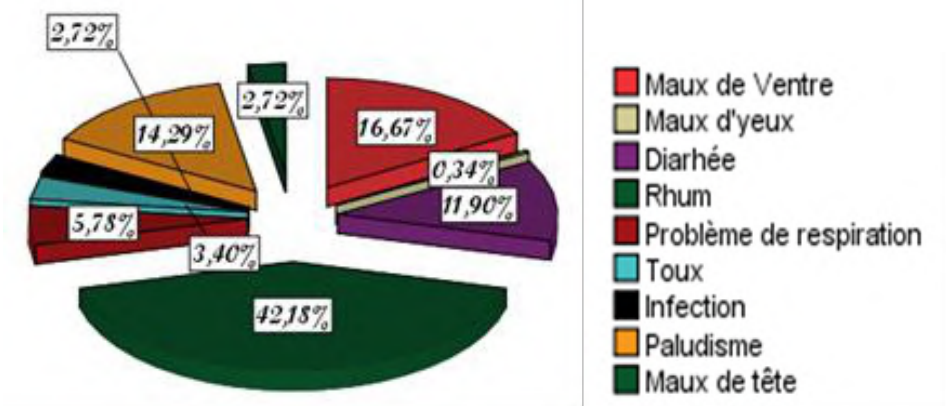

Figure 6 : Principales maladies liées à l'utilisation des produits chimiques pour la culture du coton conventionnel selon les producteurs

Source : Enquêtes de terrain

A la lecture des données de la figure 6, il ressort que les maux de ventre selon $16,67 \%$, les maux des yeux pour $0,34 \%$, la diarrhée par $11,90 \%$, les problèmes de respiration aux dires de $5,78 \%$, la toux pour $3,40 \%$, le rhume selon $2,72 \%$, les infections selon $2,72 \%$, le paludisme selon $14,29 \%$ et les maux de tête par $42,18 \%$ sont les principales maladies liées à la production du coton conventionnel selon les cotonculteurs.

La mauvaise manipulation des produits chimiques a des impacts sur la santé des populations. En effet, le non-respect des normes d'épandage d'engrais chimique pour fertiliser le sol par les cotonculteurs qui le font les mains nues, sans gants et cache-nez et le non-respect des règles élémentaires lors des traitements phytosanitaires (port de vêtement de protection adéquat, de gant, de pair de lunettes requises, de cache-nez et de bottes) exposent ces derniers à des risques d'intoxication et d'irritation. 
La pollution par les produits phytosanitaires, avec le lessivage des champs de coton traités par le ruissellement des eaux ainsi contaminées vers les cours et plans d'eau, lesquels sont encore utilisés par les communautés pour les usages domestiques (lessives, bains, boisson et préparation etc.). Une autre pratique est celle liée à la pollution des cours d'eau par le lavage des appareils de traitement phytosanitaire, les sacs d'engrais chimiques (NPK ; Urée) et les récipients ayant servi pour l'épandage dans ces eaux. Cette situation est confirmée par $66 \%$ des cotonculteurs interrogés. Le lessivage des champs de coton par les eaux de ruissellement vers les sources d'eau de consommation est source des maladies telles que les maux de tête, la dysenterie, la diarrhée, le vertige et le vomissement, etc. La planche 2 montre : (1) un cotonculteur en pleine opération de traitement phytosanitaire sans les Equipements de Protection Individuels (EPI) adéquats et (2) le prélèvement de l'eau de rivière par les populations pour divers usages domestiques.
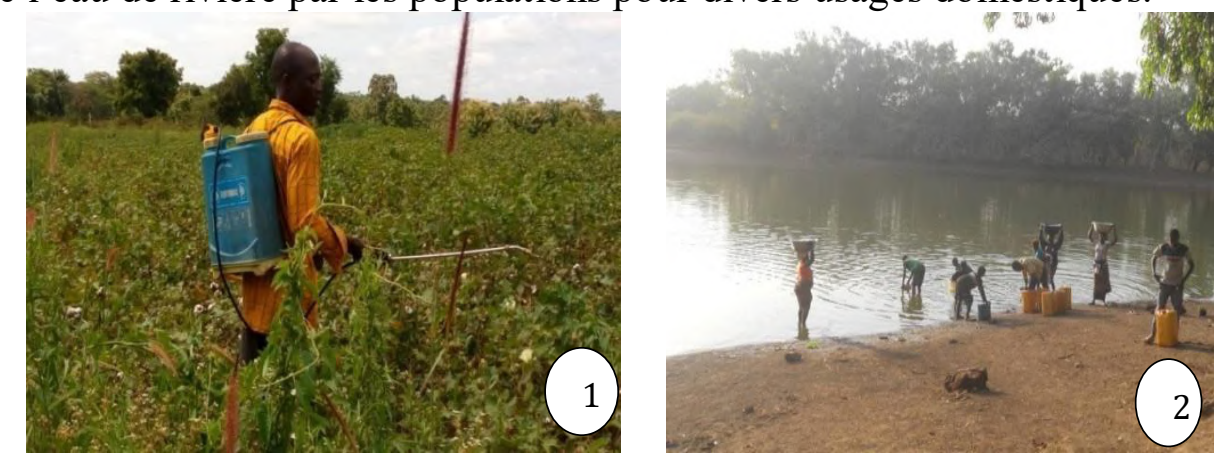

Planche 2: Traitement phytosanitaire sans EPI (photo 1) et prélèvement d'eau de rivière pour usages domestiques (photo 2)

Prise de vues : Bachabi, décembre 2016

Le manque d'accès à des informations sur les produits utilisés, l'analphabétisme, la non utilisation d'équipements de protection individuels sont entre autres des facteurs qui aggravent les risques sanitaires des cotonculteurs.

\section{Discussion}

Les engrais et pesticides chimiques utilisés pour lutter contre les parasites afin d'augmenter les rendements engendrent des conséquences sur l'environnement. Les résultats d'analyse physico-chimiques d'échantillon d'eau des puits prélevés en saison sèche et en saison pluvieuse, affichent une accumulation des métaux lourds (cadmium, plomb, cuivre, zinc). De même, les résultats d'analyse physico-chimique présentent une forte accumulation des métaux lourds comme le Cadmium (oscille entre 8,13 et $60,3 \mu \mathrm{g} / \mathrm{L}$ contre une norme de $5 \mu \mathrm{g} / \mathrm{L}$ ) et le Plomb (oscille entre 62,8 et $306 \mu \mathrm{g} / \mathrm{L}$ contre une norme de $50 \mu \mathrm{g} / \mathrm{L}$ ) dans toutes les eaux de surface prélevées en saison 
pluvieuse. Cette concentration de ces métaux lourds en saison pluvieuse est largement supérieure à la Valeur Maximale Admissible (VMA) admise par l'OMS dans tous les points d'eau analysés (eau de puits/forages concernant le cadmium, plomb, cuivre, zinc et eau de surface concernant cadmium et plomb). Cette pollution des eaux de puits/forages et des eaux de surface peut s'expliquer par une infiltration des eaux de pluie chargées des produits chimiques issus du traitement du coton vers la nappe phréatique et vers les cours d'eau du bassin versant cotonnier de l'Alibori. Ces différents résultats sont conformes à ceux rapportés par Adam Soulé (2012) et soulignent que les pesticides peuvent être entraînés dans les cours d'eau par ruissellement ou par infiltration. Ceci explique les apports des produits chimiques par le ruissellement superficiel dans les cours d'eau. Ces unités géographiques constituent des réservoirs de stockage des eaux ruisselées qui polluent les eaux des cours d'eau de la ceinture cotonnière. De même, selon (Akognongbé, 2014), la multitude de menaces importantes qui pèsent sur les ressources en eau découle des activités humaines, principalement l'usage des produits chimiques. La mauvaise manipulation des produits chimiques a des impacts sur la santé des populations. En effet, le non-respect des normes d'épandage d'engrais et le non-respect des règles élémentaires lors des traitements phytosanitaires (pas de port de vêtement de protection adéquat, pas de port de gant, pas de port de paire de lunettes requises, pas de cache-nez et pas de bottes) par les cotonculteurs, exposent ces derniers à des risques d'intoxication et d'irritation. Les résultats d'enquêtes socio-économiques montrent qu'avant et après l'utilisation des produits chimiques, aucun soin n'est pris. Les métaux lourds toxiques et autres résidus nocifs issus des divers intrants agricoles et omniprésents dans notre environnement semblent de plus en plus être impliqués dans de nombreuses pathologies chez les hommes comme chez les animaux (Boucheseiche et al., 2002). De plus, l'utilisation de ces produits est faite sans disposition adéquate de protection et sans suivi. Ces différentes pratiques provoquent chez les cotonculteurs des maladies comme les maux de ventre selon $16,67 \%$, les maux des yeux pour $0,34 \%$, la diarrhée par 11,90 $\%$, les problèmes de respiration aux dires de $5,78 \%$, la toux pour 3,40 \%, le rhume selon $2,72 \%$, les infections selon $2,72 \%$, le paludisme selon $14,29 \%$ et les maux de tête par 42,18\% et provoquent des troubles divers et variés. Ces résultats sont semblables à ceux obtenus par Kissira (2010). Selon ce dernier, les malaises dus à l'utilisation des intrants chimiques sont plus prononcés après la pulvérisation des champs de coton. Le rhume, les maux de tête, les irritations cutanées, les maux d'yeux, la toux sont les principaux problèmes de santé recensés après les traitements phytosanitaires. En effet, les cotonculteurs n'observent pas les règles élémentaires du traitement phytosanitaire. Du reste, les métaux lourds toxiques et autres résidus nocifs issus des divers intrants agricoles et omniprésents dans l'environnement 
semblent de plus en plus être impliqués dans de nombreuses pathologies chez les Hommes comme chez les animaux (Boucheseiche et al., 2002). La plupart de ces produits agissent sur le métabolisme des insectes (notamment au niveau du système nerveux) par des procédés qui sont communs à l'ensemble du règne animal, et affecte de ce fait également la santé humaine (Westerberg et al., 2017). Les symptômes connus sont divers et dépendent généralement du degré d'exposition : cela peut aller de la diarrhée au vomissement, au trouble de vue, l'irritation de la peau et des yeux, jusqu'à des symptômes beaucoup plus graves et parfois à la mort en cas d'exposition accrue ou d'ingestion (EJF, 2007). La culture du coton contribue également à la détérioration de l'état de santé des populations selon 60,91\% des cotonculteurs enquêtés. Les auteurs (Chabi Guiya, 2004 ; Kissira, 2010 ; Gomgnimbou et al., 2010 ; Orou Séko, 2012 etc.) ont également confirmé ces résultats dans leurs travaux de recherche.

\section{Conclusion}

Au terme de cette recherche, les engrais chimiques et pesticides utilisés pour lutter contre les parasites afin d'augmenter les rendements de la production du coton conventionnel dans le bassin versant de l'Alibori, engendrent des impacts négatifs sur l'environnement. En effet, les concentrations les plus importantes de métaux lourds (cadmium et le plomb) dans les eaux de surface et les puits/orages en saison pluvieuse sont liées à l'activité humaine et plus précisément aux pratiques de production du coton conventionnel dans le bassin versant de l'Alibori. Ceci s'explique par le lessivage des produits chimiques utilisés dans les champs en saison de pluie et transportés par le ruissellement superficiel des champs vers les cours d'eau. Ces unités géographiques constituent des réservoirs de stockage des eaux ruisselées que les populations utilisent pour divers usages (consommation, cuisine, abreuvage des animaux etc.). Chez l'homme, ces métaux lourds absorbés s'accumulent spécifiquement dans différents organes (les reins, le foie et même le cerveau) et provoquent des troubles divers et variés propres à chaque métal selon les enquêtes de terrain et des travaux conduits par d'autres auteurs. Des mesures de bonnes pratiques d'utilisation d'intrants chimiques méritent donc d'être vulgarisées auprès des producteurs.

\section{References:}

1. ABE, (1998) : Guide général de réalisation d'une étude d'impact sur l'environnement. Agence Béninoise pour l'Environnement, 76 pages.

2. Adam Soulé I., (2012): Pollution par les pesticides et les métaux lourds: les impacts toxicologiques de l'agriculture dans la ceinture cotonnière de Gogounou-Kandi-Banikoara, Ecole Doctorale 
Pluridisciplinaire (EDP)/FLASH/UAC. Thèse de doctorat unique, 185 p.

3. AIC (2009) : Projets d'assainissement et de relance de la filière coton au Bénin, $66 \mathrm{p}$

4. Akognongbé A. J. S (2014) : Influence de la variabilité climatique et des activités anthropiques sur les eaux de surface dans le bassin de l'ouémé a Bètèrou au Bénin. Thèse de doctorat unique, EDP/FLASH/UAC, 255p

5. Barcelona M., Gibb J.P., Helfrich J.A. et Garske E.E., (1985) : Practical guide

6. Bloundi M.K. (2005) : Etude géochimique de la lagune de Nador (Maroc oriental) : Impacts des facteurs anthropiques. Thèse de Doctorat. Ecole et Observatoire des Sciences de la Terre Centre de Géochimie de la Surface (UMR 7517) et Université Mohamed VAgdal Faculté des Sciences de Rabat, 210p.

7. Boucheseiche C., Cremille E., Pelte T., Pojer K. (2002) : Bassin Rhône Méditerranée - Corse. Guide technique $\mathrm{n}^{\circ} 7$, Pollution toxique et écotoxicologie : notion de base. Lyon, Agence de l'Eau Rhône Méditerranée - Corse, 83 p.

8. Chabi Guiya D. A. (2004) : Les facteurs de risques de santé liés à la manipulation des insecticides de coton dans la Commune de Pèrèrè, mémoire de DEA, DGAT/FLASH/UAC, 62 p.

9. Claasen H.C. (1982): Guidelines and techniques for obteining waters amples that accurately represent the water quality for an aquifer. US Geological Survey open file report 82-1024; 49 p.

10. DGEau (2008) : Atlas hydrographique du Bénin. MMEE, DANIDA, Bénin, $22 \mathrm{p}$.

11. Di Benedetto M.(1997):Cumulated Index medicus,Chicago,259 p.

12. EJF (2007): The Deadly Chemicals in Cotton, Environ- mental Justice Foundation in collaboration with Pesticide Action Network UK, London, UK. ISBN No 1-904523-10-2.

13. FAO (2007) : Interaction entre agriculture et forêt. 20ème session, Comité de l'agriculture, Rome, 25-28 avril 2007.4 p.

14. Gomgnimbou A. P. K., Savadogo W. P., Nianogo A. J. et MillogoRasolodimby J. (2010) : Pratiques agricoles et perceptions paysannes des impacts environnementaux de la cotonculture dans la province de la KOMPIENGA (Burkina Faso), Sciences \& Nature Vol.7 N ${ }^{\circ}$, pp $165-175$.

15. Hounslow A. W., (1995): Water quality data: analysis and interpretation. Boca Raton etc. : CRC Lewis.

16. IGN France (1961-1963) : Cartes topographiques, feuilles de Lomé et d'Abomey au 1/200 000. 
17. INSAE (2013): Quatrième Recensement Général de la Population et de l'Habitation (RGPH4). Direction des Etudes Démographiques, 490 p.

18. Kissira A. (2010) : les activités agricoles et la dégradation des ressources naturelles dans la Commune de ségbana au nord-est du Bénin : impacts sur la santé humaine, Thèse de Doctorat unique, UAC/FLASH/DGAT, 254 p.

19. Léopold L. B., Clarke F. E., Hanshow B. B., Bolsley J. R. (1971): A procedure for evaluating environnmental impact. Pdf $19 \mathrm{p}$.

20. Max H. (2005) : Coton équitable, première filière non alimentaire de Max Havelaar, France. Site:htm.

21. OBEPAB (2002) : Le Coton au Bénin : rapport de consultation sur le coton conventionnel et le coton biologique au Bénin, $36 \mathrm{p}$.

22. Orou Séko S. S. (2012) : Contraintes relatives à la relance de la production cotonnière dans la Commune de Banikoara, mémoire de maitrise DGEAT/FLASH/UAC, 99 p.

23. Schwartz D. (1995) : Méthodes statistiques à l'usage des médecins et des biologistes. 4è édition. Editions médicales Flammarion, Paris, 314 p.

24. Tenté A. B. (2005): Recherche sur les facteurs de la diversité floristique des versants du massif de l'Atacora: Secteur PermaToucountouna (Bénin), Thèse de Doctorat unique, UAC/FLASH/DGAT, $252 \mathrm{p}$

25. Westerberg, V., Golay, A., Houndekon, V. et Costa, L. (2017) : L'économie de la degradation de terre, le cas de la commune de Banikoara. Le coton est-il vraiment l'or blanc à Banikoara? Une publication de la Coopération Allemande et l'Initiative Economics of Land Degradation, Disponible sur www.eld-initiative.org. 